\title{
Argyrophilic Grain Pathology as a Natural Model of Tau Propagation
}

\author{
Alberto Rábano $^{\mathrm{a}, *}$, Izaskun Rodal ${ }^{\mathrm{a}}$, Raquel Cuadros ${ }^{\mathrm{b}, \mathrm{c}}$, Miguel Calero $^{\mathrm{a}, \mathrm{c}, \mathrm{d}}$, Félix Hernández ${ }^{\mathrm{b}, \mathrm{c}}$ \\ and Jesús Ávila ${ }^{\mathrm{b}, \mathrm{c}}$ \\ ${ }^{a}$ Banco de Tejidos, Fundación CIEN (FCIEN-ISCIII), Madrid, Spain \\ ${ }^{\mathrm{b}}$ Centro de Biología Molecular Severo Ochoa (CSIC-UAM), Madrid, Spain \\ ${ }^{\mathrm{c}}$ Centro de Investigación Biomédica en Red sobre Enfermedades Neurodegenerativas (CIBERNED, ISCIII), Spain \\ ${ }^{\mathrm{d}}$ Unidad de Encefalopatías Espongiformes, Unidad Funcional de Investigación en Enfermedades Crónicas, \\ (UFIEC-ISCIII), Madrid, Spain
}

Accepted 18 December 2013

\begin{abstract}
Argyrophilic grain disease (AGD) is a sporadic 4 R tauopathy that usually presents in combination with other sporadic tauopathies or with Alzheimer's disease (AD) pathology, and may contribute to dementia in older age patients. In previous studies, a detailed analysis of AGD pathology in the medial temporal lobe has been hampered by the common presence of concurrent AD changes. With the objective to assess the potentiality of AGD in research on tau propagation, here we present a study of a series of AGD postmortem cases $(n=53)$. The total series was divided in a subgroup of cases with Braak-stage $\leq \mathrm{II}(n=23)$ and a subgroup with Braak-stage $>$ II or indeterminate $(n=30)$ in order to minimize interference with AD pathology. A detailed neuropathological evaluation of the medial temporal lobe was performed at three coronal levels with Gallyas stain, and immunostains with p62, AT8, and AT100 antibodies. Western blot analysis of the entorhinal and hippocampal cortex was performed in 8 cases with a panel of anti-tau antibodies. Cases were genotyped for $A P O E$ polymorphism and for $\mathrm{H} 1 / \mathrm{H} 2$ alleles of the MAPT gene. All cases, and particularly lower Braak-stage cases, displayed a highly homogeneous pattern of involvement by argyrophilic grains and pretangles between connected regions (primarily basolateral nuclei of the amygdala, entorhinal/transentorhinal cortex, and hippocampal cortex). Staging of cases reveals progression of pathology along well-established neuroanatomical pathways. Western blot studies yielded a specific pattern of isoforms with a characteristic predominant band at $64 \mathrm{kDa}$. Genetic analysis showed a strong association with the H1 allele of the MAPT gene. AGD may thus be an optimal natural disease model for testing hypotheses related to tau propagation in human tissue.
\end{abstract}

Keywords: Argyrophilic, dementia, grains, limbic, tau, tauopathy

\section{INTRODUCTION}

Argyrophilic grain disease (AGD) is a sporadic tauopathy without specific clinical manifestations that principally involves the medial temporal lobe and the limbic region $[1,2]$. It generally presents as a postmortem neuropathological finding in two main settings: i) either combined with other neurodegenerative

\footnotetext{
*Correspondence to: Alberto Rábano, Departamento de Neuropatología y Banco de Tejidos, Fundación CIEN, c/ Valderrebollo, 5, 28031 Madrid, Spain. Tel.: +34 9138522 00; Fax: +34 91385 21 18; E-mail: arabano@fundacioncien.es.
}

diseases, most often with other sporadic tauopathies, or ii) in aged subjects with or without dementia, associated with various degrees of Alzheimer-type and/or cerebral vascular pathology $[3,4]$. The frequent combination of AGD with Alzheimer's disease (AD), particularly in the oldest-old age group of patients, may represent the effect of these two putative pathogenic factors, i.e., neurodegeneration and aging. A particularly high prevalence of AGD has been described in centenarians [5] that may reach $100 \%$ of subjects when adequate histological stains are employed [6]. Consequently, it seems that even if dementia may not 
be an ineluctable destiny for the aging human brain, AGD may finally develop in any subject that reaches an extreme old age.

Brain regions involved in AGD contain a high ratio of hyperphosphorylated tau (phospho-tau) isoforms enclosing 4 repeats (4R) of exon 10 of the $M A P T$ gene, as compared to 3-repeat (3R) isoforms [7-9]. Accordingly, AGD is classified within the $4 \mathrm{R}$ group of sporadic tauopathies, together with progressive supranuclear palsy (PSP), corticobasal degeneration (CBD), and other less common tauopathies, mainly observed in very aged patients. Additionally, AGD shares with PSP and CBD a genetic association with the H1 genotype of the MAPT gene [10]. While the characteristic cell inclusions of AGD, argyrophylic grains (AG), are specific for this entity, other cell lesions regularly found in this condition (pretangles, coiled bodies, astrocytic inclusions, and ballooned neurons) are also common in other $4 \mathrm{R}$ tauopathies. AG are 4 to $8 \mu \mathrm{m}$ oval or spindle-shape formations that can be found in high density in some nuclei of the amygdaloid complex, the entorhinal/transentorhinal cortex, and the hippocampal cortex $[3,4]$. They can be best revealed with Gallyas silver stain and with immunohistochemical techniques for phospho-tau (e.g., AT100 and AT8 anti-tau antibodies), 4 $\mathrm{R}$ tau isoforms, or p62, an ubiquitine-binding protein [11].

In rare cases, AGD presents as the only pathological substrate of a neurological syndrome, usually a cognitive disorder with or without associated behavioral symptoms. Most commonly, however, this tauopathy develops a subclinical course or may act synergistically with other concomitant pathologies (principally $\mathrm{AD}$ and vascular) in the generation of cognitive or behavioral deficits $[12,13]$. In concordance with recent lexical recommendations related to pathology of the Alzheimer-type $(14,15)$, particularly in a subclinical context, the term "argyrophilic grain pathology" (AGP) will be employed here when referring to AGD.

Along the last few years, experimental evidence has been accumulating on the potential role of phospho-tau (among other pathological proteins related to various neurodegenerative diseases) in the propagation of taurelated pathology in a prion-like manner (reviewed in [16]). Recently, the histological features of several different human tauopathies, including AGP, have been partially reproduced in transgenic animals through inoculation of brain extracts [17], a transmission model characteristic of bioassays for prion diseases.

In this study, we explore the potentiality of AGP as a natural model for research of tau transmissibility and propagation based on human tissue. Ideally, a human disease should meet the following requirements in order to be adequate for this type of research: i) a regular pattern of progression that allows staging of disease in brain tissue; ii) connectivity between involved brain areas through known neural pathways; and iii) a specific molecular signature that remains stable along disease progression. An additional feature that may bring the model closer to protein propagation in prion disease would be a polymorphic gene locus associated to disease risk and phenotype. All these features are here addressed in a series of patients with AGP from three different brain banks, where cases without relevant AD pathology have been selected for a detailed neuropathological, molecular and genetic study.

\section{MATERIALS AND METHODS}

\section{Patients}

All cases with AGP were selected from a series of 511 brains donated to three Brain Banks in Spain (Banco de Tejidos CIEN [BT-CIEN, $n=217$ ], Banco de Cerebros de la Región de Murcia [BCRM, $n=70]$, Banco de Tejidos para Investigación Neurológica / Hospital Universitario Fundación Alcorcón [BTIN/HUFA, $n=224]$ ) between 1996 and 2012. Only cases with a neuropathological diagnosis of a neurodegenerative disease and/or age at death $\geq 50$ years were included in the reference series, with a mean age of 74.9 years $( \pm 12.1$, range: $36-103)$, and a $M / F$ gender ratio of $1: 1.1$. A total 53 cases with AGP were identified (10.4\%). Associated main neuropathological diagnoses were PSP (22.6\%), AD (22.6\%), cerebrovascular disease (18.9\%), CBD (11.3\%), Lewy body pathology (Parkinson's disease or Lewy body dementia) (7.6\%), TDP-43 pathology (amyotrophic lateral sclerosis or frontotemporal lobar degenerationTDP) (7.6\%), frontotemporal lobar degeneration-tau (FTLD-tau) (5.6\%), multiple system atrophy (1.9\%), and Huntington's disease (1.9\%). Mean age at death of AGP patients was 78.7 years ( \pm 9.6 , range: $51-98)$ and total $\mathrm{M} / \mathrm{F}$ gender ratio was $1: 1.1$. Mean duration of the main neurological syndrome was 7.2 years $( \pm 5$, range: 0.3-20). Seven patients had been autopsied with a clinical suspicion of prion disease at BTIN/HUFA (a reference center for postmortem diagnosis of human prion diseases) due to a rapidly progressive neurological syndrome (mean duration: 0.97 years; range: $0.3-2$ ), and had been classified after postmortem diagnosis as vascular (3), CBD (2), or AD (2). Dementia rate for the whole series was $68 \%$, and only the $\mathrm{AD}$, 
Table 1

Main sociodemographic, clinical, neuropathological, and genetic data of the total argyrophilic grain pathology (AGP) series of patients, and of two subgroups stratified by Braak-stage. Data of the 8 patients with indeterminate Braak stage, due to high burden of concomitant tauopathy, are not included. Quantitative variables are represented as mean (SD), and qualitative variables as percentage

\begin{tabular}{|c|c|c|c|c|}
\hline & Total AGP series & Braak $\leq$ II & Braak > II & Signif. \\
\hline$n$ & 53 & 23 & 22 & \\
\hline Gender ratio (M/F) & $1: 1.1$ & $1.3: 1$ & $1: 1.75$ & NS \\
\hline Age at death (y) & $78.7(9.6)$ & $76.8(9.2)$ & $81.9(10.8)$ & NS \\
\hline Age at onset (y) & $71(9.1)$ & $70.2(10.6)$ & $73.1(8.6)$ & NS \\
\hline Disease duration (y) & $7.22(5)$ & $7.1(5.9)$ & $7.4(4.4)$ & NS \\
\hline Dementia $(\%)$ & 78.3 & 68.4 & 84.2 & NS \\
\hline AGP stage & $2.36(0.7)$ & $2.3(0.7)$ & $2.4(0.7)$ & NS \\
\hline Tauopathies (\%) & 33 & 39 & 27 & NS \\
\hline Lewy pathology (\%) & 27 & 26 & 27 & NS \\
\hline Other neurodegen. disease $(\%)$ & 16 & 30 & 9 & NS \\
\hline Vascular $(\%)$ & 18 & 30 & 5 & $p<0.05$ \\
\hline Brain weight (g) & $1063(122)$ & $1100(126)$ & $1059(116)$ & NS \\
\hline Neuritic plaques (CERAD) & $1.00(1.18)$ & $0.26(0.54)$ & $2.00(1.19)$ & $p<0.01$ \\
\hline Braak stage & $2.91(1.64)$ & $1.61(0.72)$ & $4.27(1.12)$ & $p<0.01$ \\
\hline Braak stage (MTL) & $2.65(1.54)$ & $1.50(0.89)$ & $3.93(1.12)$ & $p<0.01$ \\
\hline Amyloid- $\beta$ hippocampus & $2.00(1.67)$ & $0.83(1.34)$ & $3.33(0.89)$ & $p<0.01$ \\
\hline NIA score & $0.94(1.16)$ & $0.17(0.39)$ & $2.00(1.02)$ & $p<0.01$ \\
\hline Amyloid angiopathy & $0.48(0.85)$ & $0.19(0.6)$ & $0.95(0.99)$ & $p<0.01$ \\
\hline Small vessel disease & $1.66(1.01)$ & $1.38(1.02)$ & $1.95(0.86)$ & $p=0.062$ \\
\hline Hippocampal sclerosis (\%) & 17 & 8.7 & 22.7 & NS \\
\hline TDP-43 (+) (\%) & 28.6 & 15.8 & 50 & $p<0.05$ \\
\hline$A P O E \& 4(\%)$ & 17.6 & 16.6 & 21.4 & NS \\
\hline$M A P T \mathrm{H} 1$ & 97.1 & 100 & 92.9 & NS \\
\hline
\end{tabular}

CBD, FTLD-tau, and Huntington's disease subgroups did not include any patient without dementia (Table 1).

\section{Neuropathology}

All brain donations were processed according to a common postmortem protocol [18]. A rapid neuropathological autopsy was performed upon call by the donor's proxies (mean postmortem interval, $4.5 \mathrm{~h}$ ). In conventional-risk donation cases, immediately after extraction, the right half of the brain was sliced and frozen, while the left half was fixed by immersion in phosphate-buffered $4 \%$ formaldehyde for at least 3 weeks. High-risk autopsies for suspected CJD were performed following international recommendations. In these cases, fresh tissue blocks from the right frontal and parietal lobes and the ipsilateral cerebellar hemisphere were obtained for freezing. All frozen tissue was stored at $-80^{\circ} \mathrm{C}$. After fixation, a full neuropathological study was performed in the left half brain. Neuropathological diagnosis and staging of all disease entities, including AGP, was performed according to published consensus criteria [15]. Various neuropathological variables related to $\mathrm{AD}$, vascular, Lewy and TDP pathologies, as well as the presence of hippocampal sclerosis, were recorded for full classification of cases.
Assessment of AGP was performed in paraffin sections (left brain hemisphere) at three coronal (anterior-posterior) levels of the medial temporal lobe: i) Level 1 (L1) corresponds to a mid-coronal plane of the amygdala including the adjoining entorhinal and perirhinal cortices; ii) Level 2 (L2) includes the anterior segment of the hippocampus, the entorhinal cortex and the medial and lateral banks of the collateral sulcus; and iii) Level 3 (L3) is homologous to L2 at a posterior coronal plane, and includes the anterior segment of the body of the hippocampus together with the adjoining cortices, including the collateral sulcus. Consecutive paraffin sections of each block were stained either with Gallyas, or immunostained with AT8, AT100 (1 : 100, Innogenetics, Gent, Belgium), and p62 (Thermo Fisher Scientific Inc., Waltham, Massachusetts) primary antibodies. An ABC method (Pierce Antibodies, Thermo Fisher Scientific Inc., Waltham, Massachusetts) was employed for the amplification of immunohistochemical staining, and the slides were revealed with DAB. A semiquantitative ( $0-3$ score) assessment of the frequency of AG, pretangles (pNFT), and neurofibrillary tangles (NFT) was performed in multiple areas at each coronal Level: nuclei of the amygdala (L1), entorhinal/transentorhinal cortex (L1-2), perirhinal cortex (L1-3), subiculum (L2-3), CA1 (L2-3), CA2-3 (L23 ), and dentate gyrus (L3). While AG were assessed 
with Gallyas and both phospho-tau and p62 immunostains, pNFT were assessed only with phospho-tau immunohistochemistry, and NFT were evaluated only on Gallyas' stained sections. AG frequency was also assessed in several extratemporal regions: posterior hypothalamus, nucleus accumbens, anterior insular and cyngular cortex, and brainstem tegmentum. Staging of AGP was performed according to Thal et al. [13]. Additional L2 and L3 sections were immunostained for amyloid- $\beta$ (clone 6F/3D, Dako Laboratories) and TDP-43 (clone 2E2/E3, Abnova, Thermo Fisher Scientific Inc., Waltham, Massachusetts).

\section{Western blot analysis}

Selective sampling of the entorhinal and of the hippocampal cortex in frozen tissue was performed in each of 8 brains of patients with Braak stage $\leq$ II ( 4 cases of BTCIEN and 4 cases of BCRM brain bank). For this analysis 3 PSP, 2 CBD, 1 Parkinson's disease, 1 multiple system atrophy, and 1 vascular case were selected. Brain cell extracts were isolated by homogenizing the brain tissue in ice-cold buffer $(1: 10 \mathrm{w} / \mathrm{v})$ consisting of $10 \mathrm{mM}$ Tris pH7.4, $1 \mathrm{mM}$ EGTA, $0.1 \mathrm{M} \mathrm{NaCl}$ and $10 \%$ sucrose plus phosphatase inhibitors $(10 \mathrm{mM} \mathrm{NaF}$, $1 \mathrm{mM}$ sodium orthovanadate), and protease inhibitors ( $2 \mathrm{mM}$ PMSF, $10 \mu \mathrm{g} / \mathrm{ml}$ aprotinin, $10 \mu \mathrm{g} / \mathrm{ml}$ leupeptin, and $10 \mu \mathrm{g} / \mathrm{ml}$ pepstatin). Tau aggregates from the brain of the patients were isolated as previously described for PHF-tau from AD patients [19, 20]. Extracts for western blot analysis were prepared by homogenizing the isolated tau aggregates samples. The samples were homogenized at $4{ }^{\circ} \mathrm{C}$ and protein content determined by Bradford method (BioRad). Total protein $(10 \mu \mathrm{g})$ was electrophoresed on $8 \%$ SDS-PAGE gel and transferred to a nitrocellulose membrane. The experiments were performed using the following primary antibodies: 7.51, PHF-1, Tau 4R, and exon 2. Anti-tau 7.51 $(1: 100)$ recognizes a region containing a part of the microtubule-binding domain of human and murine tau, and is a kind gift of Claude M. Wischik, Newcastle University, UK. PHF-1 (1:100), an antibody that reacts with tau when Ser396 and Ser404 are phosphorylated, was a kind gift of Peter Davies, Albert Einstein University, NY, USA. Anti-tau clone 1E1/A6 (1:500), specific for reacting with tau $4 \mathrm{R}$ isoforms was from Merck Millipore, Billerica, Massachusetts. Finally, exon-2 $(1: 100)$ antibody was raised against human tau peptide comprising the residues 55 to 67 (numbering of the longest human tau isoform [20]), and it was obtained through Abyntek Biopharma S.L., (Derio, Spain) for our laboratory (R. Cuadros et al., unpub- lished data, 2011). The membranes were incubated with the antibody at $4{ }^{\circ} \mathrm{C}$ overnight in $5 \%$ nonfat dried milk. A secondary HRP-conjugated goat anti-mouse (Gibco, Life Technologies Corp., Carlsbad, California) followed by ECL detection reagents (Amersham, GE Healthcare Bio-Sciences, Piscataway, New Jersey) were used for immunodetection.

\section{Genotyping}

Total DNA was isolated from peripheral blood or cerebral tissue following standard procedures. Genotyping of MAPT rs1052553 polymorphism, which discriminates between MAPT $\mathrm{H} 1$ and $\mathrm{H} 2$ haplotypes, was determined using TaqMan $^{\circledR}$ probes (C_7563736_10 assay, Applied Biosystems) according to manufacturer's instructions. APOE genotyping (rs429358 and rs7412) was performed by Real-Time PCR [21]. Published data from $\mathrm{H} 1$ and $\mathrm{H} 2$ prevalence in the normal Spanish population were used as a comparative group $[22,23]$, as well as data of the Vallecas Project (an ongoing longitudinal study on aging currently developed at Fundación CIEN, Madrid, Spain), corresponding to 249 subjects without neurological disease (age range: $68-85 \mathrm{y} ; \mathrm{M} / \mathrm{F}$ ratio, $1: 1.87)$. Control data for the analysis of $A P O E$ were also obtained from the Vallecas Project $(n=1147)$. The odds ratio (OR) and the significance value were obtained through the $\chi^{2}$ statistic with the aid of the GraphPad Prism v4 program. HardyWeinberg equilibrium of the genotype distributions of control populations was determined by means of the analysis of the distribution of observed versus expected genotype frequencies. The level of significance was based on a $\chi^{2}$ distribution with one degree of freedom.

\section{RESULTS}

\section{Neuropathology}

All cases with AGP displayed the full histological picture, with the presence of a moderate to high density of AG and pNFT in the basolateral nuclei of the amygdala and the anterior segments of the entorhinal/transentorhinal cortex and CA1 sector of the hippocampal cortex. Both in the amygdala and the hippocampal cortex, AG co-distributed closely with pNFT, while in the parahippocampal cortical regions, a high density of AG in layers II and III (and to a lesser extent in the upper segment of layer V) were associated more loosely to pNFT, mainly in layers III and $\mathrm{V}$ (upper sublayer). A marked gradient of lesional density can be easily discerned from the transentorhi- 


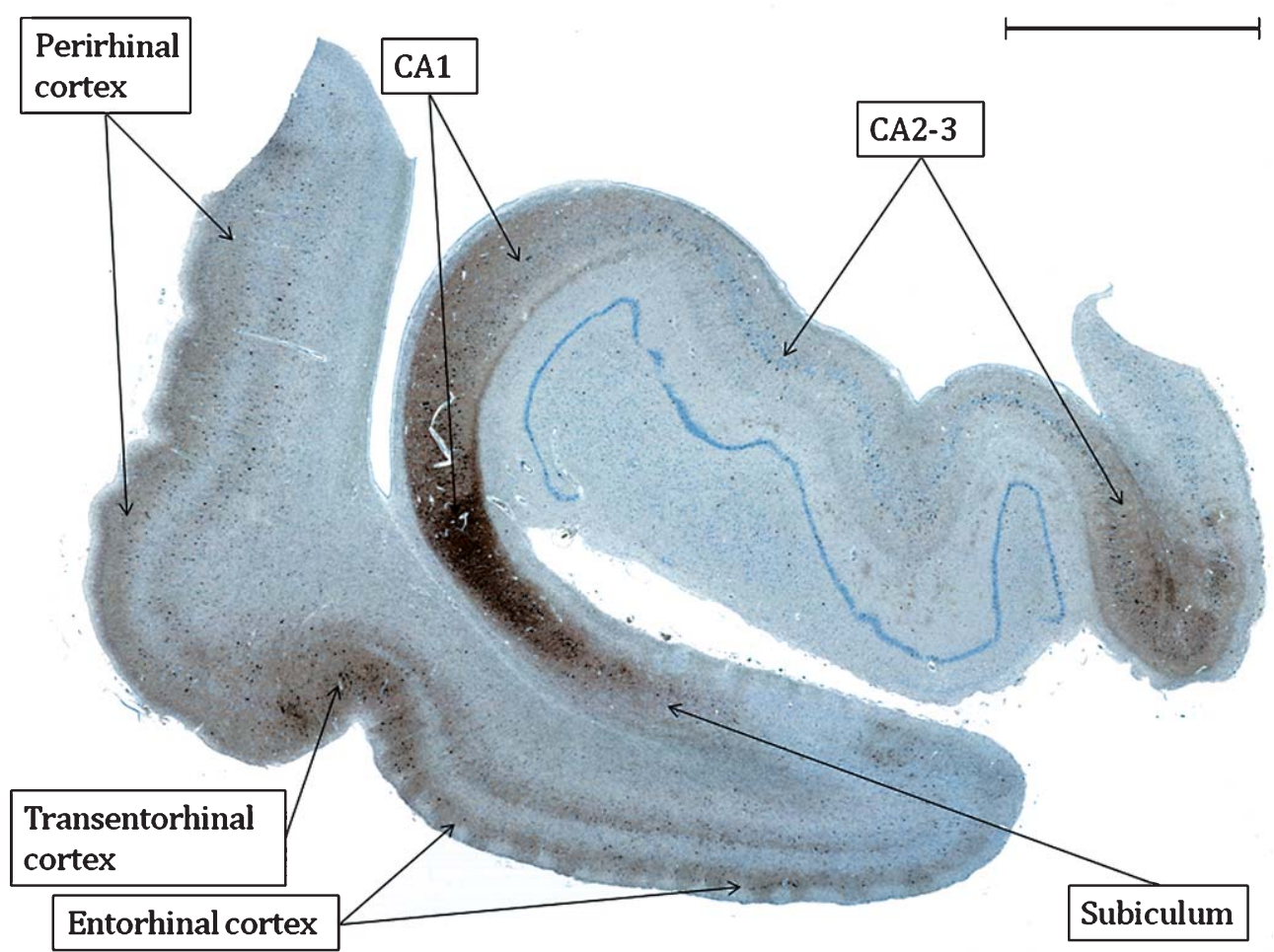

Fig. 1. Very low magnification image of a coronal section at Level 2 (anterior hippocampus) of Patient 22 (AGP + Vascular + Parkinson's disease) immunostained with AT8 antibody. Note the intense staining of distal CA1 sector and transentorhinal cortex with a double gradient at each side. Relevant staining of perirhinal cortex indicates stage 3 AGP. Scale bar $=0.5 \mathrm{~cm}$.
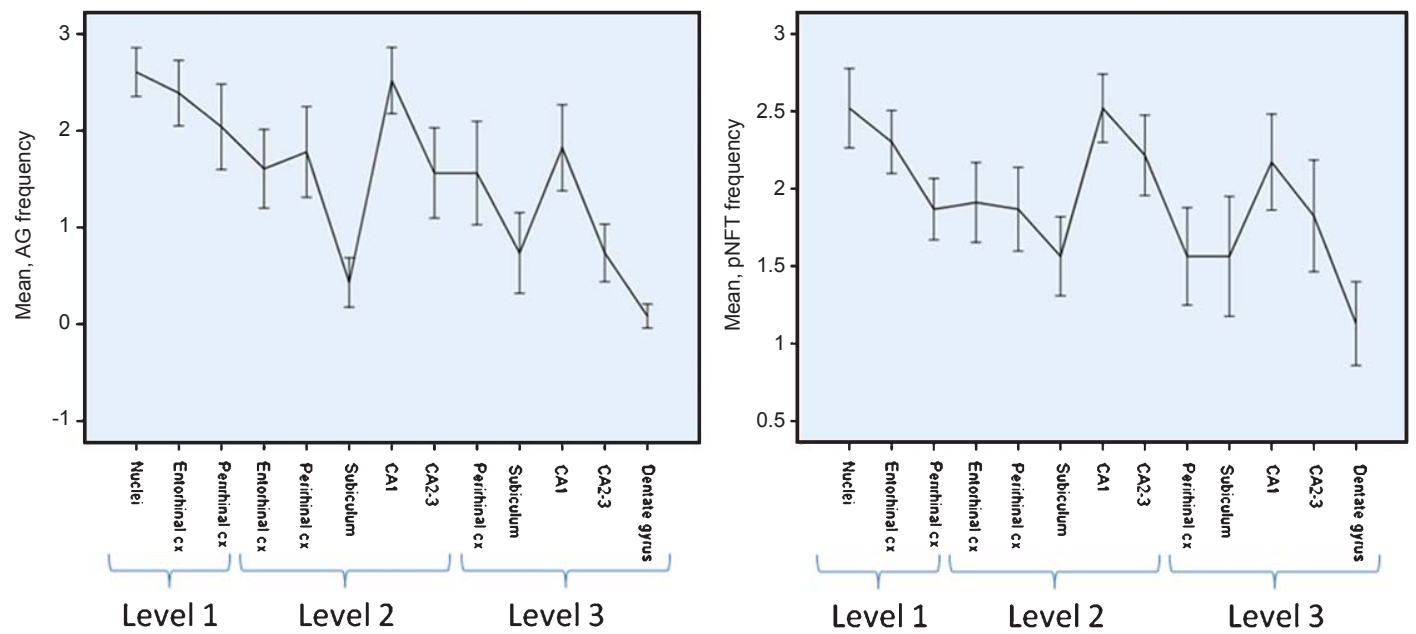

Fig. 2. Lesion profile for AG (left) and pNFT (right) for all regions assessed at the three coronal levels considered. Points connected by graph lines represent the mean value for the frequency of each lesion at each evaluated area. Bars represent the $95 \%$ confidence interval. Note the existence of a peak-region and a valley-region for each coronal level. Homology between levels and regions is highlighted by the consecutive representation of all three levels.

nal cortex in both directions, laterally and medially, and from the subiculum-CA1 junction towards CA2-3 (Fig. 1). Other involved areas in the medial temporal lobe, e.g., perirhinal cortex and CA2-3 sectors, showed more variable frequencies of AG and pNFT, and other characteristic lesions, like coiled bodies, astrocytic 


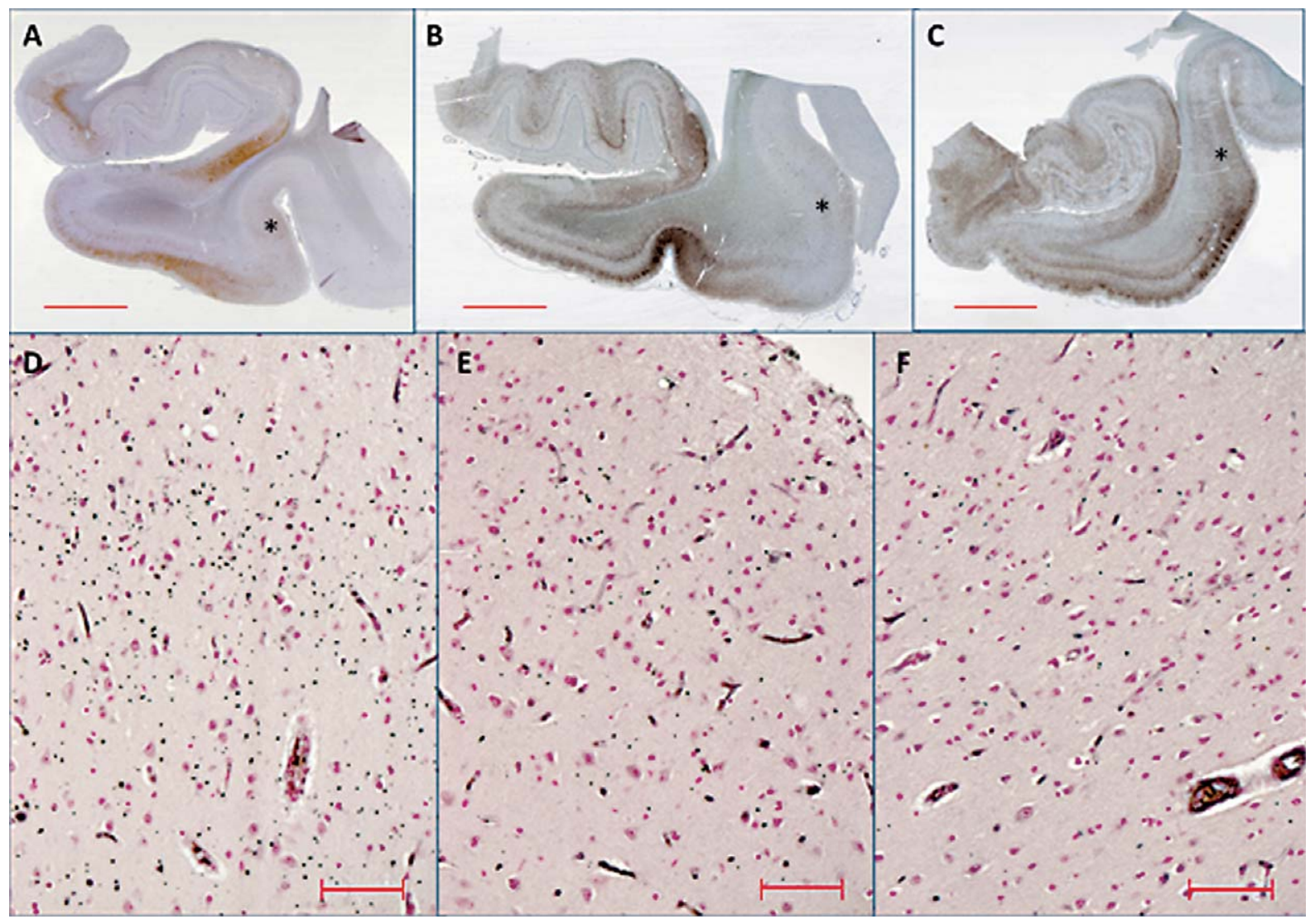

Fig. 3. A-C) Very low magnification images of coronal sections at Level 2 (anterior hippocampus) immunostained with AT8 antibody, illustrating the distribution and intensity of AGP lesions in stage 1 (A), stage 2 (B), and stage 3 (C) cases. Note the evident extension of pathology to the perirhinal cortex $(*)$ in stage 3 . Scale bar $=0.5 \mathrm{~cm}$. D-F) Low magnification images of Gallyas-stained sections of transentorhinal (D), perirhinal (E), and lower temporal (F) cortex in a stage 3 AGP patient. Note the progressive decrease in AG density along the medial to lateral axis. Scale bar $=100 \mu \mathrm{m}$.
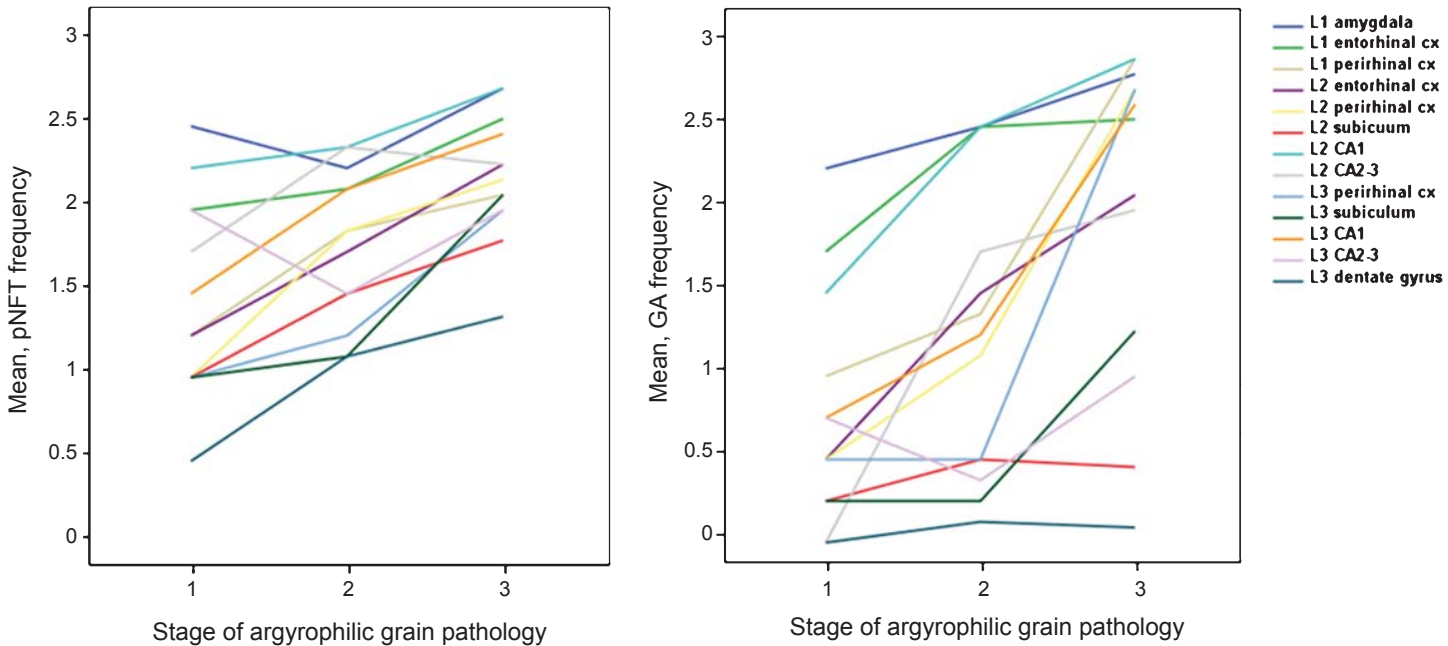

Fig. 4. Mean value of pNFT (left) and AG (right) frequency at all evaluated regions for cases stratified by AGP stage. L1, Level 1; L2, Level 2; L3, Level 3 . 


\begin{tabular}{|c|c|c|c|c|c|c|c|c|}
\hline \multirow{2}{*}{ Patient } & \multirow{2}{*}{$\begin{array}{c}\text { Level } 1 \\
\text { Amygdala }\end{array}$} & \multirow{2}{*}{\begin{tabular}{c|} 
Level 2 \\
Ant. hippoc. \\
\end{tabular}} & \multirow{2}{*}{\begin{tabular}{|c} 
Level 3 \\
Post. hippoc.
\end{tabular}} & \multirow{2}{*}{ Stage } & \multicolumn{2}{|c|}{ Level 2} & \multicolumn{2}{|c|}{ Level 3} \\
\hline & & & & & CA1 & CA2-3 & CA1 & CA2-3 \\
\hline 5 & & & & 3 & & & & \\
\hline 3 & & & & 3 & & & & \\
\hline 25 & & & & 3 & & & & \\
\hline 53 & & & & 3 & & & & \\
\hline 50 & & & & 3 & & & & \\
\hline 47 & & & & 3 & & & & \\
\hline 18 & & & & 3 & & & & \\
\hline 48 & & & & 3 & & & & \\
\hline 22 & & & & 3 & & & & \\
\hline 30 & & & & 2 & & & & \\
\hline 10 & & & & 2 & & & & \\
\hline 27 & & & & 3 & & & & \\
\hline 38 & & & & 2 & & & & \\
\hline 51 & & & & 2 & & & & \\
\hline 43 & & & & 2 & & & & \\
\hline 15 & & & & 2 & & & & \\
\hline 7 & & & & 2 & & & & \\
\hline 44 & & & & 2 & & & & \\
\hline 40 & & & & 1 & & & & \\
\hline 2 & & & & 1 & & & & \\
\hline 13 & & & & 2 & & & & \\
\hline 17 & & & & 1 & & & & \\
\hline
\end{tabular}

Fig. 5. Schematic representation of the frequency of AG in the perirhinal cortex of patients with Braak stage $\leq$ II (left). White color equals 0 score, whereas grey color of increasing intensity represents scores 1 to 3 . Cases have been arranged in decreasing order of intensity. AG density at the hippocampal cortex of patients ordered according to the intensity of perirhinal pathology (right) shows similar though less regular gradients.

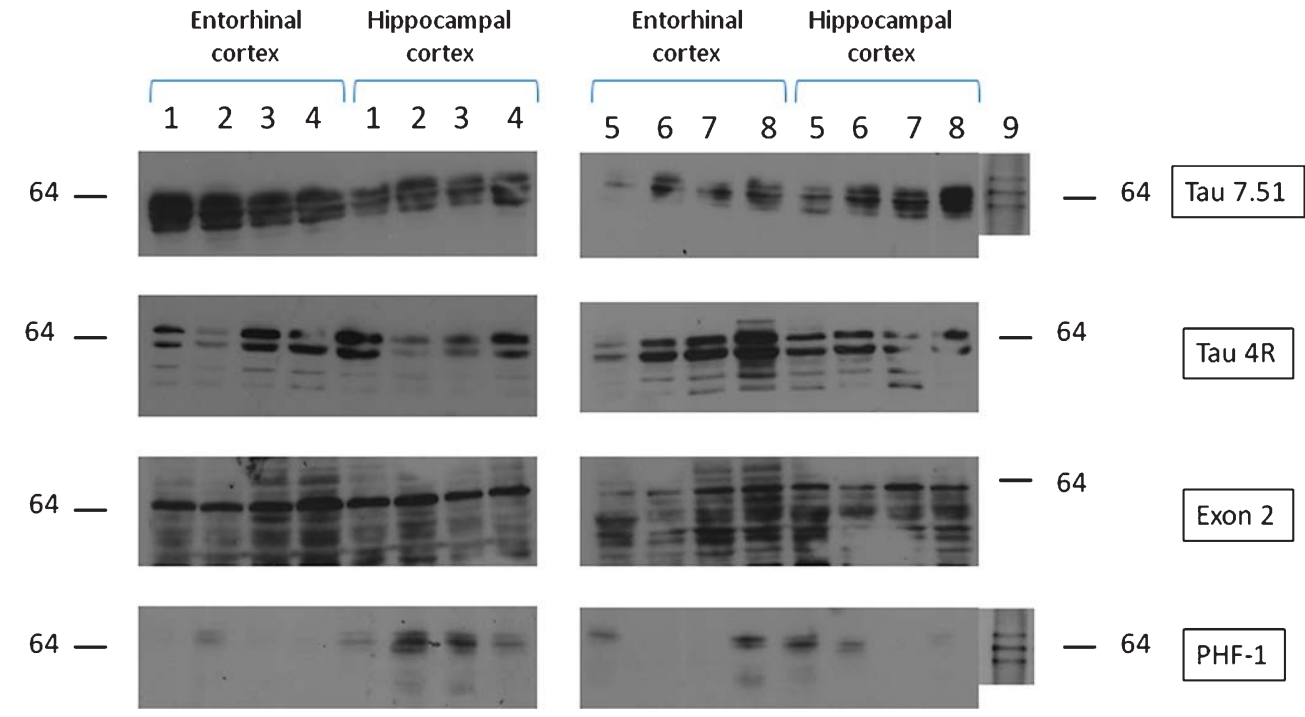

Fig. 6. Western blot analysis of tau protein in several patients with Braak stage $\leq$ II. The left panels correspond to 4 patients of the BT-CIEN brain bank (1: AGD + PSP; 2: AGD + vascular; 3: AGD + PSP; 4: AGD + multiple system atrophy), and the right panels represents 4 patients from the BCRM bank (5: AGD + PSP; 6: AGD+Parkinson's disease; 7: AGD + CBD; 8: AGD +CBD). Samples of the entorhinal and hippocampal cortex of the same patients are shown in consecutive groups. Lane 9 represents a patient with pure AD (Braak VI) for comparison. See main text for description of findings. 
Table 2

Distribution of genotypes obtained in the total argyrophilic grain pathology (AGP) series after analysis of the APOE polymorphism and the $\mathrm{H} 1 / \mathrm{H} 2$ alleles of MAPT. Control groups: for $A P O E$ analysis, CONT 1, Vallecas Project (see main text); for MAPT, CONT 1, Vallecas Project (see main text), CONT 2, Ref. [22], CONT 3, Ref. [23]. OR has been calculated with respect to the total CONT $(1+2+3)$ group

\begin{tabular}{|c|c|c|c|c|c|c|}
\hline APOE $\varepsilon 4$ carriers & $(-)$ & $(+)$ & OR ( $p$ value $)$ & & & \\
\hline AGP & 28 & 6 & $1.15(0.76)$ & & & \\
\hline CONT 1 & 958 & 179 & - & & & \\
\hline $\mathrm{APOE} \varepsilon 2$ carriers & $(-)$ & $(+)$ & OR ( $p$ value) & & & \\
\hline AGP & 32 & 2 & $0.54(0.39)$ & & & \\
\hline CONT 1 & 1027 & 120 & - & & & \\
\hline \multirow[t]{2}{*}{ MAPT } & \multicolumn{3}{|c|}{ Genotype (n) } & \multicolumn{2}{|c|}{ Alleles (\%) } & Association ( $\mathrm{H} 1$ versus $\mathrm{H} 2)$ \\
\hline & $\overline{\mathrm{H} 1 / \mathrm{H} 1}$ & $\mathrm{H} 1 / \mathrm{H} 2$ & $\mathrm{H} 2 / \mathrm{H} 2$ & H1 & $\mathrm{H} 2$ & OR ( $p$ value $)$ \\
\hline AGP & 28 & 5 & 1 & $61(89.7 \%)$ & $7(10.3 \%)$ & $3.45(0.001)$ \\
\hline CONT 1 & 110 & 118 & 21 & $338(67.9 \%)$ & $160(32.1 \%)$ & - \\
\hline CONT 2 & 98 & 81 & 15 & $277(71.4 \%)$ & $111(28.6 \%)$ & - \\
\hline CONT 3 & 170 & 120 & 17 & $460(74.9 \%)$ & $154(25.1 \%)$ & - \\
\hline CONT $(1+2+3)$ & 378 & 319 & 53 & $1075(71.7 \%)$ & $425(28.3 \%)$ & - \\
\hline
\end{tabular}

inclusions, and ballooned neurons, presented a less regular density of distribution, though limited in all cases to areas involved by AG and pNFT. Extratemporal AG were detected in $70 \%$ of cases, most frequently in the posterior hypothalamus (lateral tuberous nucleus and adjoining areas) (53\%) and in the nucleus accumbens of the striatum (43\%). Other involved regions were the anterior insular and cyngular cortex and, less frequently, the brainstem tegmentum. Figure 2 shows the mean lesion profile for AG (Fig. 2A) and pNFT (Fig. 2B) displayed along the anterior-posterior axis $(\mathrm{L} 1 \longrightarrow 2 \longrightarrow 3$ ). At each level, a mean peak is observed in the nuclei of the amygdala (L1), and CA1 sector (L2 and L3), respectively, with a decreasing gradient toward the entorhinal and perirhinal cortices, and toward more proximal sectors of the hippocampal cortex. A significant correlation was observed between AG and pNFT at all levels (Spearman test, data not shown). After staging for AGP, $46 \%$ of cases were classified as stage 3,40\% as stage 2, and $14 \%$ as stage 1. Differences between stages can be highlighted (Fig. 3) by a significant increase in the density of pathology at the entorhinal/transentorhinal and CA1 regions (stage 1 versus stage 2 ) and by the progressive extension of pathology toward the perirhinal cortex (stage 2 versus stage 3 ). Virtually all areas evaluated at all levels showed progressive increase of AG and pNFT frequency with increasing AGP stage (Fig. 4). No significant correlation or association was found between AGP stage and any other clinical or neuropathological classification variables. A higher proportion of extratemporal pathology was observed in stage 3 cases versus stage 1 or 2 (non significant, $p=0.09$ ).

In order to analyze more accurately AGP without an interference of concomitant $\mathrm{AD}$ pathology, cases were stratified with respect to Braak stage II, and cases with lower Braak stages were selected for further analysis. A total of 23 cases were identified with Braak stage $\leq$ II, in 22 additional cases Braak stage was $>$ II, and in 8 cases (various tauopathies) staging could not be precisely established, in most cases due to concomitant tau pathology. Significant differences between both groups were mainly limited to histological features related to AD pathology (Table 1). Additionally, a higher score for small vessel pathology was observed in the higherBraak group (non significant, $p=0.06$ ), and this group also displayed a significantly higher frequency of TDP$43(+)$ inclusions in the hippocampus. No correlation was observed between Braak stage and AGP stage. As the perirhinal cortex at all coronal levels seemed to represent a well-defined lateral front of progression of AGP, all cases within the lower-Braak group were arranged in decreasing order of AG frequency at this cortical area (Fig. 5, left). This arrangement, closely correlated with AGD stage, reveals a progressive increment of AG along an anterior-posterior axis (from L1 to L3), particularly in stage 2 and 3 cases, and along a medial to lateral axis, as can be observed in stage 2 and 3 cases in Fig. 3. The corresponding arrangement of AG data for CA1 and CA2-3 at L2 and L3, keeping the previous ordering by perirhinal cortex data (Fig. 5, right), yields a less regular though evident pattern of progression along an anterior-posterior and a CA1 to CA2-3 axis.

\section{Western blot analysis}

Samples of entorhinal and hippocampal cortex of 8 brains included in the lower-Braak stage group showed in all cases a high load of protein and a common pattern 
of bands with several anti-tau antibodies (Fig. 6). An anti-4R antibody shows an intense double band consistent with a 4R tauopathy, whereas one of these bands, at $64 \mathrm{k}$, stains intensely with an antibody specific for exon 2, and is the predominant band revealed by PHF1 , a monoclonal antibody that recognizes tau isoforms phosphorylated at serines 396 and 404. From these findings, it can be inferred that the double band recognized by the $4 \mathrm{R}$ anti-tau contains predominantly the two $4 \mathrm{R}$ tau isoforms containing either exon 2 $(64 \mathrm{kDa})$ or no additional exon $(62 \mathrm{kDa})$, and that the predominant hyperphosphorylated tau isoform in AGP corresponds to the $4 \mathrm{R}$ isoform that contains exon 2 and lacks exon 3.

\section{Genotyping}

Table 2 depicts the relative frequency of APOE $\varepsilon 4$ and $\varepsilon 2$ alleles among the AGP group, as well as the distribution of the $\mathrm{H} 1$ and $\mathrm{H} 2$ alleles of the MAPT gene, as compared to the respective control groups. No association was detected between AGP and any of the APOE alleles. However, a strong association $(\mathrm{OR}=3.45, p<0.001)$ was observed with the $\mathrm{H} 1$ allele of $M A P T$.

\section{DISCUSSION}

Over the last few years, it has become manifest that tauopathies, similarly to other neurodegenerative diseases, may spread through the central nervous system in a prion-like manner. This pathogenic model would imply the propagation of the pathological tau protein, or at least of its characteristic posttranslational changes, along neural pathways involved in disease. In contrast to prion diseases, however, no evidence of infectivity or of natural transmission between individuals has been found for the pathological tau protein. As is the case with tau pathology of $\mathrm{AD}$, most sporadic tauopathies involve extensive brain regions, both cortical and subcortical, and a variety of neuronal and glial tau inclusions [24, 25]. This expansive spread of pathology seems to be highly difficult to trace in terms of cell-to-cell transmission of tau, and only the early stages of neurofibrillary degeneration in Alzheimer-type pathology can suggest the actual neuron populations and pathways involved [26]. In contrast, argyrophilic grain disease, here referred to as argyrophilic grain pathology (AGP) due to its mostly subclinical course, exhibits a short number of closely related tau-positive inclusions, and affects a more localized brain region, limited principally to the medial temporal lobe. Consequently, in a series of patients with a neuropathological diagnosis of AGP, this study focused on all features that may support cellto-cell propagation of pathological tau protein in this entity.

The first set of evidence relates to the topographical pattern of distribution of the characteristic cell lesions of AGP. All cases of the series presented here share a common homogeneous pattern of distribution of AG and pNFT: basolateral nuclei of the amygdala and anterior segments of the entorhinal/transentorhinal cortex and CA1 cortex, respectively. Progression of AGP is evidenced by a higher density of pathology in these regions, by spreading of AG and pNFT to other nuclei of the amygdala, by the extension of pathology along the parahippocampal cortices toward posterior and lateral areas, as well as by spreading of lesions to more proximal sectors of the hippocampal cortex. Previous studies have suggested this multidirectional advance of AGP, and extended it to further spreading to other limbic extratemporal regions [27, 28]. In our series, stage 3 cases showed a nonsignificant trend toward a higher extratemporal spread, as previously reported [27]. However, this extension seems to be too early and variable in some cases for its use as a criterion for staging. No association of higher stages to combined tauopathies and no correlation with age were found, as reported previously [27].

When the series of patients with AGP was stratified in a lower- and a higher-Braak stage groups so as to analyze AGP in the medial temporal lobe without associated tau pathology of the Alzheimer-type, the only significant difference between the groups with respect to neuropathological classification data, other than $\mathrm{AD}$ pathology-related variables, was a higher proportion of TDP-43 (+) inclusions in higher Braak cases. Previously, a high proportion of TDP-43 immunoreactive lesions has been described in AGP [29], though in our series it is mainly limited to patients with a higher burden of AD pathology.

Areas involved by AGP in the medial temporal lobe are connected by well-known pathways. The entorhinal/transentorhinal cortex is directly connected with the CA1 and subiculum through the minor perforant pathway [30]. The presence of an intensity gradient in both areas is also observed in early stages of $\mathrm{AD}$ tau pathology, and has been adduced as an argument in favor of a possible role of connectivity in this disease [31]. The entorhinal/transentorhinal cortex is also well connected to the basolateral 
nuclei of the amygdala and to the perirhinal cortex. There also exists well-known connectivity between the extratemporal areas of involvement by AGP with the entorhinal/transentorhinal cortex and/or the amygdala (basolateral nuclei). Finally, progression of pathology along continuous cortical areas (e.g., entorhinal cortex or CA1 sector of the hippocampus) could be channeled through lateral connectivity between contiguous cortical domains.

In samples of the entorhinal and hippocampal cortex a predominant band of phospho-tau at $64 \mathrm{kDa}$ was found on western blot analysis. This band is also revealed by specific antibodies that recognize 4R isoforms and exon 2, suggesting, as previously reported, a predominance in AGP of the tau isoform bearing 4 repeats of exon 10 and one copy of exon 2 [32]. Previous molecular studies of AGP have emphasized the similarity of the patterns of isoforms obtained to those of other 4R tauopathies (PSP and CBD) [7-9, 33]. However, the predominance of this isoform may be a specific feature of AGP, present in our series in cases with and without an associated $4 \mathrm{R}$ tauopathy (Fig. 6), as previously reported. Some features characteristic of CBD, as compared to PSP, have also been observed although they have not been confirmed by other authors [34].

No association with APOE alleles was found, in concordance with one previous study based on a large series of cases [35]. In contrast, a strong association was identified with the $\mathrm{H} 1$ allele of MAPT gene, as observed previously [10], and as is well-established for other 4R tauopathies [36]. The number of patients carrying at least one $\mathrm{H} 2$ allele $(n=6)$ is too low to obtain information of a possible association of this uncommon genetic feature to a specific phenotypic trait. However, it may be relevant to note that $50 \%$ of cases in this group presented clinically as a rapidly progressive neurological syndrome.

In summary, AGP displays a highly homogeneous pattern of distribution and progression of pathology along several regions of the medial temporal lobe with known connectivity between them and with extratemporal areas of involvement. AGP also presents a molecular signature (pattern of predominant tau isoforms and strong association to the $\mathrm{H} 1$ allele of $M A P T$ ), consistent with the putative propagation of pathological tau species as a basis for the expansion of pathology. Consequently, AGP may be an adequate natural model for research in tau propagation. The perirhinal cortex seems to be an optimal region where to study the actual front of advance of this particular tauopathy in human tissue.

\section{ACKNOWLEDGMENTS}

This work was supported by grants from the Spanish Plan Nacional (SAF2011-24841), CIBERNED, the Joint Programming for Neurodegenerative Diseases (Demtest Project), and Reina Sofia Foundation. We wish to express our acknowledgement to E. Gómez (BT-CIEN), V. Martínez, L. Peyres (BCRM), and A. B. Rebolledo (BTIN / HUFA) for their technical work, and to C. Guerrero (BTIN / HUFA) for her assistance in neuropathological diagnosis.

Authors' disclosures available online (http://www. j-alz.com/disclosures/view.php?id=2069).

\section{REFERENCES}

[1] Braak H, Braak E (1987) Argyrophilic grains: Characteristic pathology of cerebral cortex in cases of adult onset dementia without Alzheimer changes. Neurosci Lett 76, 124-127.

[2] Braak H, Braak E (1989) Argyrophilic grain disease: Frequency of occurrence in different age categories and neuropathological diagnostic criteria. J Neural Transm 105, 801-819.

[3] Tolnay M, Clavaguera F (2004) Argyrophilic grain disease: A late-onset dementia with distinctive features among tauopathies. Neuropathology 24, 269-283.

[4] Ferrer I, Santpere G, van Leeuwen FW (2008) Argyrophylic grain disease. Brain 131, 1416-1432.

[5] Ding ZT, Wang Y, Jiang YP, Yoshida M, Mimuro M, Inagaki T, Iwase T, Hashizume Y (2006) Argyrophilic grain disease: Frequency and neuropathology in centenarians. Acta Neuropathol 111, 320-328.

[6] Pham CT, de Silva R, Haik S, Verny M, Sachet A, Forette B, Lees A, Hauw JJ, Duyckaerts C (2011) Tau-positive grains are constant in centenarians' hippocampus. Neurobiol Aging 32, 1296-1303.

[7] Togo T, Cookson N, Dickson DW (2002) Argyrophilic grain disease: Neuropathology, frequency in a dementia brain bank and lack of relationship with apolipoprotein E. Brain Pathol 12, 45-52.

[8] Tolnay M, Sergeant N, Ghestem A, Chalbot S, De Vos RA, Jansen Steur EN, Probst A, Delacourte A (2002) Argyrophilic grain disease and Alzheimer's disease are distinguished by their different distribution of tau protein isoforms. Acta Neuropathol 104, 425-434.

[9] Zhukareva V, Shah K, Uryu K, Braak H, Del Tredici K, Sundarraj S, Clark C, Trojanowski JQ, Lee VM (2002) Biochemical analysis of tau proteins in argyrophilic grain disease, Alzheimer's disease, and Pick's disease: A comparative study. Am J Pathol 161, 1135-1141.

[10] Conrad C, Vianna C, Schultz C, Thal DR, Ghebremedhin E, Lenz J, Braak H, Davies P (2004) Molecular evolution and genetics of the Saitohin gene and tau haplotype in Alzheimer's disease and argyrophilic grain disease. J Neurochem 89, 179188.

[11] Scott IS, Lowe JS (2007) The ubiquitin-binding protein p62 identifies argyrophilic grain pathology with greater sensitivity than conventional silver stains. Acta Neuropathol 113, 417420.

[12] Josephs KA, Whitwell JL, Parisi JE, Knopman DS, Boeve BF, Geda YE, Jack CR Jr, Petersen RC, Dickson DW (2008) 
Argyrophilic grains: A distinct disease or an additive pathology? Neurobiol Aging 29, 566-573.

[13] Thal DR, Schultz C, Botez G, Del Tredici K, Mrak RE, Griffin WS, Wiestler OD, Braak H, Ghebremedhin E (2005) The impact of argyrophilic grain disease on the development of dementia and its relationship to concurrent Alzheimer's disease-related pathology. Neuropathol Appl Neurobiol 31, 270-279.

[14] Dubois B, Feldman HH, Jacova C, Cummings JL, Dekosky ST, Barberger-Gateau P, Delacourte A, Frisoni G, Fox NC, Galasko D, Gauthier S, Hampel H, Jicha GA, Meguro K, O'Brien J, Pasquier F, Robert P, Rossor M, Salloway S, Sarazin M, de Souza LC, Stern Y, Visser PJ, Scheltens P (2010) Revising the definition of Alzheimer's disease: A new lexicon. Lancet Neurol 9, 1118-1127.

[15] Montine TJ, Phelps CH, Beach TG, Bigio EH, Cairns NJ, Dickson DW, Duyckaerts C, Frosch MP, Masliah E, Mirra SS, Nelson PT, Schneider JA, Thal DR, Trojanowski JQ, Vinters HV, Hyman BT, National Institute on Aging, Alzheimer's, Association (2012) National Institute on Aging-Alzheimer's Association guidelines for the neuropathologic assessment of Alzheimer's disease: A practical approach. Acta Neuropathol 123, 1-11.

[16] Soto C (2012) Transmissible proteins: Expanding the prion heresy. Cell 149, 968-977.

[17] Clavaguera F, Akatsu H, Fraser G, Crowther RA, Frank S, Hench J, Probst A, Winkler DT, Reichwald J, Staufenbiel M, Ghetti B, Goedert M, Tolnay M (2013) Brain homogenates from human tauopathies induce tau inclusions in mouse brain. Proc Natl Acad Sci U S A 110, 9535-9540.

[18] Martínez-Martín P, Avila J, Research Unit AD, Investigators (2010) Alzheimer Center Reina Sofia Foundation: Fighting the disease and providing overall solutions. J Alzheimers Dis 21, 337-348

[19] Greenberg SG, Davies P (1990) A preparation of Alzheimer paired helical filaments that displays distinct tau proteins by polyacrylamide gel electrophoresis. Proc Natl Acad Sci U S A 87, 5827-5831.

[20] Goedert M, Spillantini MG, Potier MC, Ulrich J, Crowther RA (1989) Cloning and sequencing of the cDNA encoding an isoform of microtubule-associated protein tau containing four tandem repeats: Differential expression of tau protein mRNAs in human brain. EMBO J 8, 393-399.

[21] Calero O, Hortiguela R, Bullido MJ, Calero M (2009) Apolipoprotein E genotyping method by real time PCR, a fast and cost-effective alternative to the TaqMan and FRET assays. J Neurosci Methods 183, 238-240.

[22] Bullido MJ, Aldudo J, Frank A, Coria F, Avila J, Valdivieso F (2000) A polymorphism in the tau gene associated with risk for Alzheimer's disease. Neurosci Lett 278, 49-52.

[23] Combarros O, Rodero L, Infante J, Palacio E, Llorca J, Fernández-Viadero C, Peña N, Berciano J (2003) Agedependent association between the Q7R polymorphism in the Saitohin gene and sporadic Alzheimer's disease. Dement Geriatr Cogn Disord 16, 132-135.

[24] Williams DR, Lees AJ (2009) Progressive supranuclear palsy: Clinicopathological concepts and diagnostic challenges. Lancet Neurol 8, 270-279.

[25] Kouri N, Whitwell JL, Josephs KA, Rademakers R, Dickson DW (2011) Corticobasal degeneration: A pathologically distinct 4R tauopathy. Nat Rev Neurol 7, 263-272.

[26] Braak H, Del Tredici K (2011) Alzheimer's pathogenesis: Is there neuron-to-neuron propagation? Acta Neuropathol 121, 589-595.

[27] Saito Y, Ruberu NN, Sawabe M, Arai T, Tanaka N, Kakuta Y, Yamanouchi H, Murayama S (2004) Staging of argyrophilic grains: An age-associated tauopathy. J Neuropathol Exp Neurol 63, 911-918.

[28] Thal DR, Capetillo-Zarate E, Galuske RA (2008) Tracing of temporo-entorhinal connections in the human brain: Cognitively impaired argyrophilic grain disease cases show dendritic alterations but no axonal disconnection of temporoentorhinal association neurons. Acta Neuropathol 115, 175-183.

[29] Fujishiro H, Uchikado H, Arai T, Hasegawa M, Akiyama H, Yokota O, Tsuchiya K, Togo T, Iseki E, Hirayasu Y (2009) Accumulation of phosphorylated TDP-43 in brains of patients with argyrophilic grain disease. Acta Neuropathol 117, 151158.

[30] Amaral D and Lavenex P. (2007) Hippocampal neuroanatomy. In The Hippocampus Book, Andersen P, Morris R, Amaral T, Bliss T, O'Keefe J eds. Oxford UP, New York, pp. 37-114.

[31] Lace G, Savva GM, Forster G, de Silva R, Brayne C, Matthews FE, Barclay JJ, Dakin L, Ince PG, Wharton SB, MRC-CFAS (2009) Hippocampal tau pathology is related to neuroanatomical connections: An ageing population-based study. Brain 132(Pt 5), 1324-1334.

[32] Rábano A, Cuadros R, Calero M, Hernández F, Avila J (2013) Specific profile of tau isoforms in argyrophylic grain disease. J Exp Neurosci 7, 51-59.

[33] Dickson DW, Kouri N, Murray ME, Josephs KA (2011) Neuropathology of frontotemporal lobar degeneration-tau (FTLD-tau). J Mol Neurosci 45, 384-389.

[34] Arai T, Ikeda K, Akiyama H, Nonaka T, Hasegawa M, Ishiguro K, Iritani S, Tsuchiya K, Iseki E, Yagishita S, Oda $\mathrm{T}$, Mochizuki A (2004) Identification of amino-terminally cleaved tau fragments that distinguish progressive supranuclear palsy from corticobasal degeneration. Ann Neurol 55, 72-79.

[35] Togo T, Cookson N, Dickson DW (2002) Argyrophilic grain disease: Neuropathology, frequency in a dementia brain bank and lack of relationship with apolipoprotein E. Brain Pathol 12, 45-52.

[36] Avila J, Lucas JJ, Perez M, Hernandez F (2004) Role of tau protein in both physiological and pathological conditions. Physiol Rev 84, 361-384. 